\title{
The Triangular Technique for Abdominal Myofascial Plication
}

\author{
MOHAMED S.M. HASSAN, M.D.* and WAEL NAEEM THABET GHEBERY, M.D.** \\ The Department of Plastic Surgery, Faculty of Medicine, Fayoum* and Cairo** Universities, Egypt
}

\begin{abstract}
Introduction: Abdominoplasty has two fundamental principles: First: Removal of excess fat and skin from the abdominal wall. Second: Narrowing of the waistline. Myofascial plication is a fundamental part of abdominoplasty that is done by most surgeons to reinforce the weakened abdominal wall and to produce a narrow waistline. Despite many techniques were depicted for abdominal wall plication, there is no general agreement among plastic surgeons about the ideal technique. In this article, the authors demonstrate and evaluate the triangular technique for myofascial plication.
\end{abstract}

Patients and Methods: Between January 2019 and February 2020, the authors applied the triangle plication technique on 19 women with rectus muscle diastasis who underwent anterior abdominal wall plication as a part of conventional abdominoplasty. The patients were followed-up for 7-12 months post-operative. The results were assessed mainly by measuring the reduction in the waist to hip ratio, the patients' satisfaction on a scale of 0 to 10 , and by an external observer using the Global Aesthetic Improvement SCORE (GAIS).

Results: Patients' satisfaction were graded as excellent in 95\% of cases and the GAIS ranged between scores 4-5 in $95 \%$ of cases with $17.3 \%$ average reduction in the ratio of waist to hip measures and $19.3 \%$ average reduction of the vertical length of the rectus sheath.

Conclusion: Abdominal myofascial triangular plication is a simple technique that results in improvement of the trunk contour with a narrower waistline, facilitates tension-free closure of abdominoplasty incision, and with high patients' satisfaction rates.

Key Words: Abdominoplasty - Myofascial - Plication Triangle technique.

\section{INTRODUCTION}

Abdominoplasty aims to remove the abdominal wall excess fat and skin, and to narrow the waistline $[1,2]$.

Most surgeons perform myofascial plication as a fundamental part of abdominoplasty for the strengthening of the weakened abdominal wall, especially for middle-aged women who had multiple pregnancies, and to reach a narrow waistline $[2,3]$.

Numerous plication techniques were depicted as vertical, horizontal, fusiform, curved, "H"- shaped and a combination of horizontal and vertical techniques as in crossbow plication techniques [49]. However, to date, there is no general agreement among plastic surgeons about the ideal technique for myofascial plication as abdominoplasty patients ask for a more narrow waistline, from the author point of view, they are affected by social media beauty trends, and this made abdominoplasty continuously develop, improve, and become increasingly complicated $[\mathbf{1 , 1 0}]$.

The increase in patients' aesthetic demands has led the authors to try the triangular plication technique to meet the patients' expectations. And in this article, the authors demonstrate and evaluate the triangular technique of abdominal myofascial plication which shortens the vertical length of the rectus sheath and narrows the waistline.

\section{PATIENTS AND METHODS}

This prospective clinical study was conducted from January 2019 to February 2020, where 19 women with rectus diastasis were operated on for abdominoplasty using the triangular myofascial plication. The age of the patients ranged from 25 to 56 years with an average age of 44 years.

The inclusion criteria were: All women with abdominal laxity and rectus diastasis who presented for an abdominoplasty and were requesting a betterdefined waistline and had no plans for future pregnancy.

The Exclusion criteria were: All male patients, women with a good abdominal musculature, women not sure or had plans for future pregnancy, women with BMI above $30 \mathrm{~kg} / \mathrm{m}^{2}$, smokers, chronic respiratory problems, chronic gaseous distention, positive risks for wound healing or thromboembolism and those with a contraindication for general anesthesia.

History and Examination: Preoperative detailed history taking with the general and local examination of all patients was performed besides routine 
laboratory investigations and abdominal ultrasound examination with documentation of the state of anterior abdominal muscles, any hernias, and rectus diastasis (RD). After that, using a measuring tape, the waist circumference was measured at the narrowest point of the natural waist or a point $2.5 \mathrm{~cm}$ superior to the umbilicus if the natural waist is illdefined. And the hip circumference was measured at the widest point of the buttocks, and waist to hip ratio was calculated and documented.

Surgical details: After preoperative markings and photography in a standing position, abdominoplasty was done under general anesthesia in a supine position.

After induction of the anesthesia and muscle relaxation, a urinary bladder catheter was inserted and the intra-bladder pressure was measured by using the patient's own urine with the Foley's manometer following the Desie et al., [11] technique as a simple estimate for the intra-abdominal pressure. The anesthetist measured the peak airway pressure when the muscles were fully relaxed.

Using the conventional method, a transverse incision was set at the lower abdominal crease and was extended laterally as required to include the excess skin. Then, the dermo-adipose flap was elevated, by sharp dissection, as far as the xiphoid in a cone-shaped fashion and no dissection was performed laterally to the lateral margin of recti above the umbilicus except for cases who had developed central skin bulge after plication. Periumbilical hernia, found in 9 cases, was managed by excision of the sac, reducing the content, and closing the defect longitudinally in 2 layers.

Myofascial plication was carried out using the triangular plication technique by first marking a triangle, using methylene blue, between 3 fixed points: The Xiphoid and both Anterior Superior Iliac Spines (ASIS) as shown in Fig. (1A \& 1C) and Photo (1A). The apex and the mid-base of the triangle were marked by two size 0 silk simple sutures and the distance between them was measured and recorded.

The supra-umbilical plication, down to the umbilicus, was performed by approximating the upper halves of the lateral sides of the triangle towards the mid-line, Photo (1B), using size 0 Nylon interrupted triangle mattress suture, described by Ferreira et al. [12], starting from midline passing for around $3 \mathrm{~cm}$ in oblique lateral cranial direction to one rectus sheath then from the corresponding point on the opposite rectus sheath passing in the medial caudal direction back to the midline and ending in a triangular shape and securing the knot. The triangle mattress sutures, as required, were used to shift the umbilicus up towards the new position on the muscles at the midpoint between the xiphoid process and suprapubic ridge, and shortening of the vertical length of the rectus sheath. Then, a second continuous enforcing size 2/0 Vicryl suture layer was applied.

The infra umbilical part of plication was performed by approximating the lower halves of the lateral sides and the base of the triangle towards a line extending between the umbilicus and ASIS on each side, starting from ASIS towards the umbilicus by using a continuous size 0 Nylon suture. Then, a second interrupted simple enforcing size 2/0 Vicryl suture layer was applied, (Photo 1C). Plication is completed when the three plication lines meet around the umbilicus as shown in Photo (1D) and Fig. (1B \& 1D). The abdominal wall was well strengthened in all cases, and neither extra sutures nor a synthetic mesh was required for further strengthening.
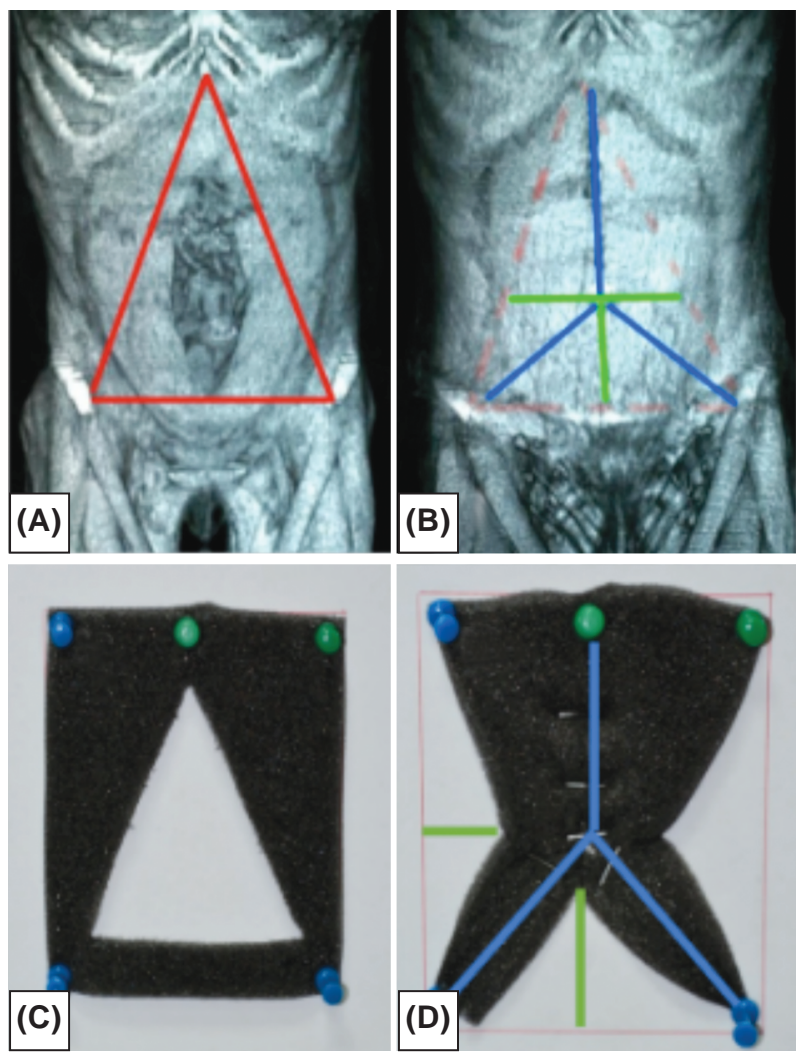

Fig. (1): (A \& C) A schematic diagram of the intraoperative marking of a triangle, between the Xiphoid and both anterior superior iliac spines, was drawn in solid red lines. (B \& D) The blue lines are a schematic presentation of the effect after completion of the triangular plication on the vertical and horizontal spans with the green vertical and horizontal lines represent the reduction of vertical and horizontal spans respectively from the original triangle represented in dotted red lines. (A \& B are adapted from Wikimedia.org under Creative Commons Attribution-Share Alike 3.0 Unported license with modification as cropping and colored line drawings). 

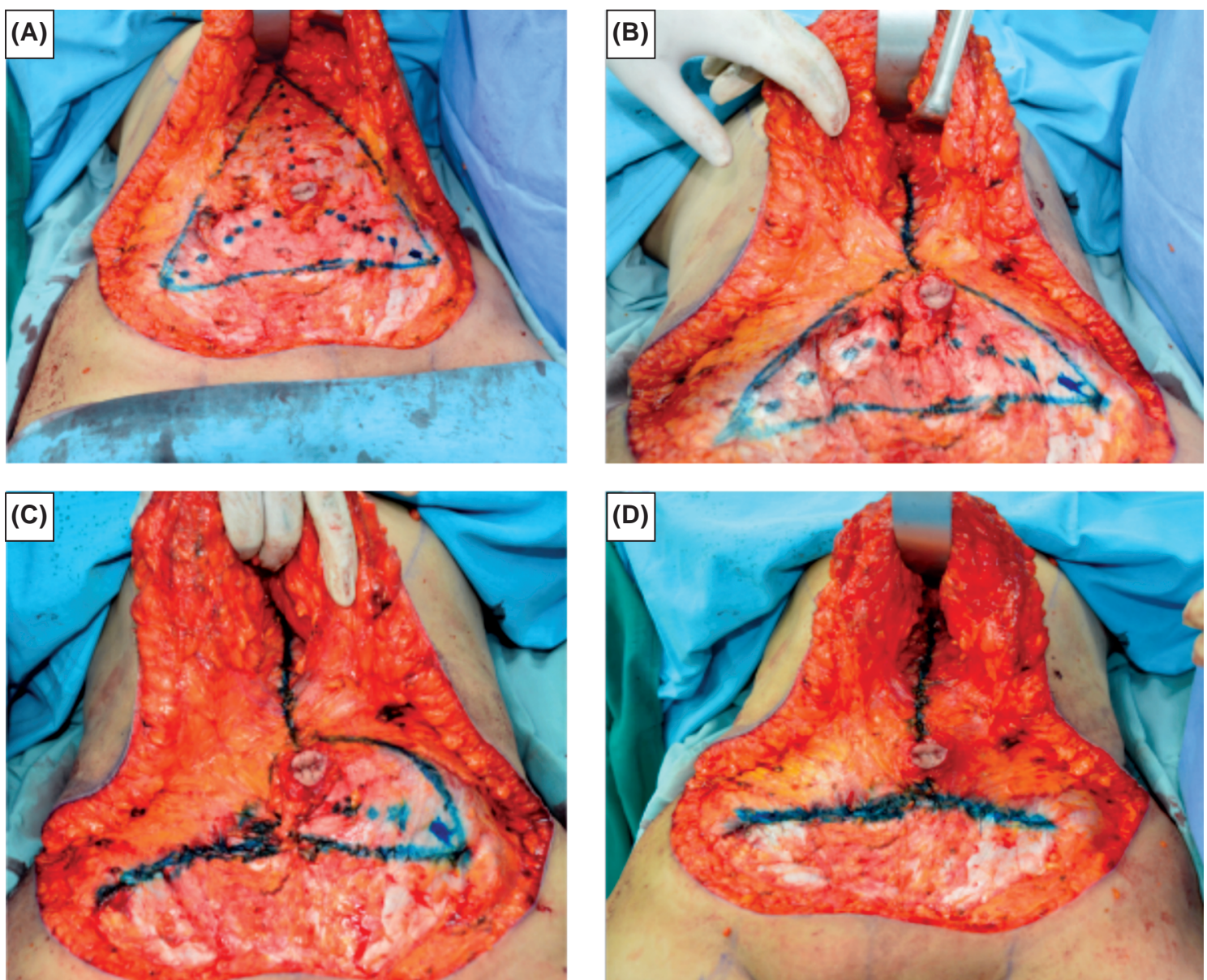

Photo (1): (A) Intraoperative marking of the triangle between the Xiphoid and both anterior superior iliac spines. (B) The endpoint of the supra-umbilical segment of the plication ending at the umbilicus and showing no extra lateral dissection beyond the lateral edge of the recti. (C) The completed right side of infra umbilical plication from the anterior superior iliac spine towards the umbilicus showing flattening of the right side compared with the left side. (D) The completion of the triangle plication with the three lines of plication are meeting at the umbilicus.

After plication was completed, the distance between the silk sutures was re-measured and recorded, then the silk sutures were removed. The anesthetist recorded the peak airway pressure and the intra-bladder pressure.

The excess skin was evaluated and excised, followed by meticulous hemostasis.

The authors did not use liposuction with any of the patients, but only trimmed the subcutaneous fat around the incision in the dermo-adipose flap to create a periumbilical hollowness before the umbilicus was transposed to its new location and sutured.

The vertical shortening of the rectus sheath facilitated shifting of the dermo-adipose flap downward and the Scarp's fascia and subcutaneous tissue were approximated using interrupted size 3/0 Vicryl sutures. And finally, lower abdominal incision was closed in layers using continuous intradermal sutures after placement of subcutaneous suction drains.

The total duration of the procedure from induction of anesthesia to extubating the patient, the duration from the skin incision to the last skin suture, and the time consumed to execute the triangular myofascial plication were all recorded.

Postoperatively, the patients wore pressure garments for 6 weeks and were discharged home on the second day, and the drain was removed in the clinic when less than $30 \mathrm{ml}$. The patients followed-up regularly every week for the first month, then once monthly thereafter to detect any post- 
operative complications and patients' satisfaction, waist to hip ratio, and photography.

The results were graded both by: First, the patients' satisfaction on a scale of 0 to 10 by summating patients' response to (1- The position of the umbilicus, 2- Narrowing of the waist, 3The excess skin or abdominal wall bulging, 4- The dog ears and scar, 5- Persistent pain or discomfort) with 2 points for each item if satisfied, 1 point for each item if neutral, and 0 points for each item if disappointed. Second, by external observers for (the waist to hip ratio, level and position of the umbilicus, excess skin or bulging) using the Global Aesthetic Improvement Score (GAIS) that was described by De Lorenzi et al., [13] [a five-point relative improvement score (1- Worsened relative to the initial condition, 2- Unaltered and the same as before intervention, 3- Condition improved but touches up are required, 4- The condition much improved but not optimum, 5- Exceptionally improved with excellent results)].

\section{RESULTS} (2-5).

Some patients' results are represented in Photos

All the patients had abdominal laxity and rectus diastasis. Nine patients (36.7\%) had periumbilical hernias.

The total duration of the procedure from the induction of anesthesia to extubating the patient ranged from 155 to 180 minutes (average 166 minutes). And the duration from the skin incision to the last skin suture ranged from 111 to 139 minutes (average 126 minutes). And the time consumed to execute the triangular myofascial plication ranged from 13 to 19 minutes (average 14 minutes) which was more or less that of the conventional midline plication in our hands.

The Intra-abdominal pressure before the plication ranged between 4.5 and $6.5 \mathrm{CM} \mathrm{H}_{2} \mathrm{O}$ (average $5.4 \mathrm{~cm} \mathrm{H}_{2} \mathrm{O}$ ), and at the end of plication ranged between 8.6 and $10.3 \mathrm{~cm} \mathrm{H}_{2} \mathrm{O}$ (average of $9.5 \mathrm{~cm}$ $\mathrm{H}_{2} \mathrm{O}$ ). The peak airway pressure before plication ranged between 18 and $22 \mathrm{~cm} \mathrm{H}_{2} \mathrm{O}$ (average $19.8 \mathrm{~cm}$ $\mathrm{H}_{2} \mathrm{O}$ ) and at the end of myofascial plication ranged between 22- and $26 \mathrm{~cm} \mathrm{H}_{2} \mathrm{O}$ (average $24.5 \mathrm{~cm}$ $\mathrm{H}_{2} \mathrm{O}$ ).

The vertical length, between the 2 silk sutures at the apex and mid-base of the triangle, before triangular plication ranged between 26 and $39 \mathrm{~cm}$ (average $30.4 \mathrm{~cm}$ ) and after triangular plication ranged between 20.5 and $29.5 \mathrm{~cm}$ average $24.5 \mathrm{~cm}$ with $19.4 \%$ average reduction in the vertical length.

The uninterrupted follow-up of the patients ranged between 2-4 months (average 3.3 months). Then, follow-up of patients was interrupted and affected by lockdown and curfew of COVID-19 pandemic for 4 months. During this time, the authors followed-up with the patients, by phone for any complications and satisfaction. After that, follow-up was resumed in the clinic until 12 months postoperatively. The average duration of the total follow-up was 9.5 months (range 7-12 months).

Seroma developed in one case (5\%), which were treated successfully by repeated aspiration over 2 weeks. Three patients (15.8\%) had early post-operative tightness for which patients were advised to move bending slightly forward and setting in a 45 degrees position and tightness improved over two weeks. One case (5\%) complained of hypertrophy of the scar, which improved over time by massage and Derma Roller. There were no other or severe complications.

The average preoperative ratio of waist to hip was 1.03 (range 0.89-1.18). And the average postoperative ratio of the waist to hip was 0.83 (range $0.71-0.92$ ) with a $17.3 \%$ average reduction of the ratio of waist to hip measures (range 9.2\%$24.8 \%$ ).

Patients' satisfaction responses were graded as excellent in 18 cases (95\%), and good in 1 case $(5 \%)$. There was no change in the patients' satisfaction rate as the results were maintained after 12 months from surgery as shown in Photo (5).

The average Global Aesthetic Improvement Score (GAIS) ranged between scores 4-5 in 18 cases $(95 \%)$ and was a score 3 in one case $(5 \%)$. 

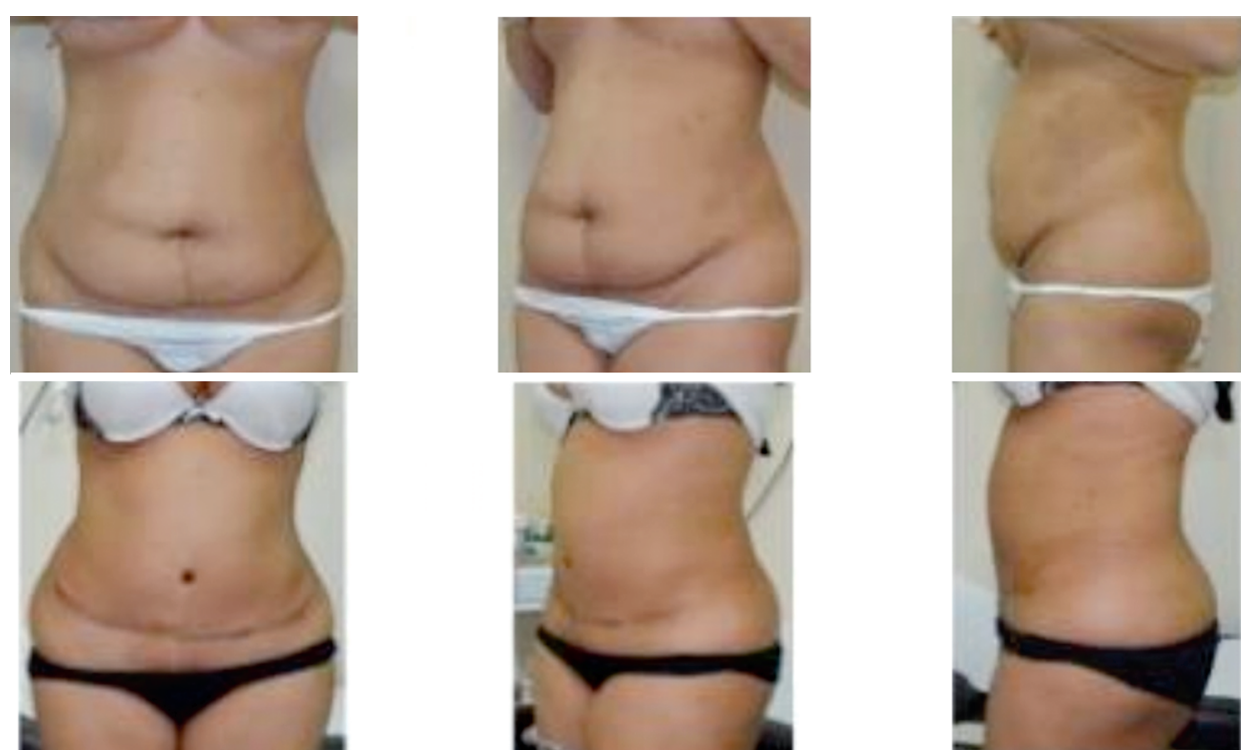

Photo (2): Case 1: Photos before and 4 months after abdominoplasty using myofascial triangular plication.
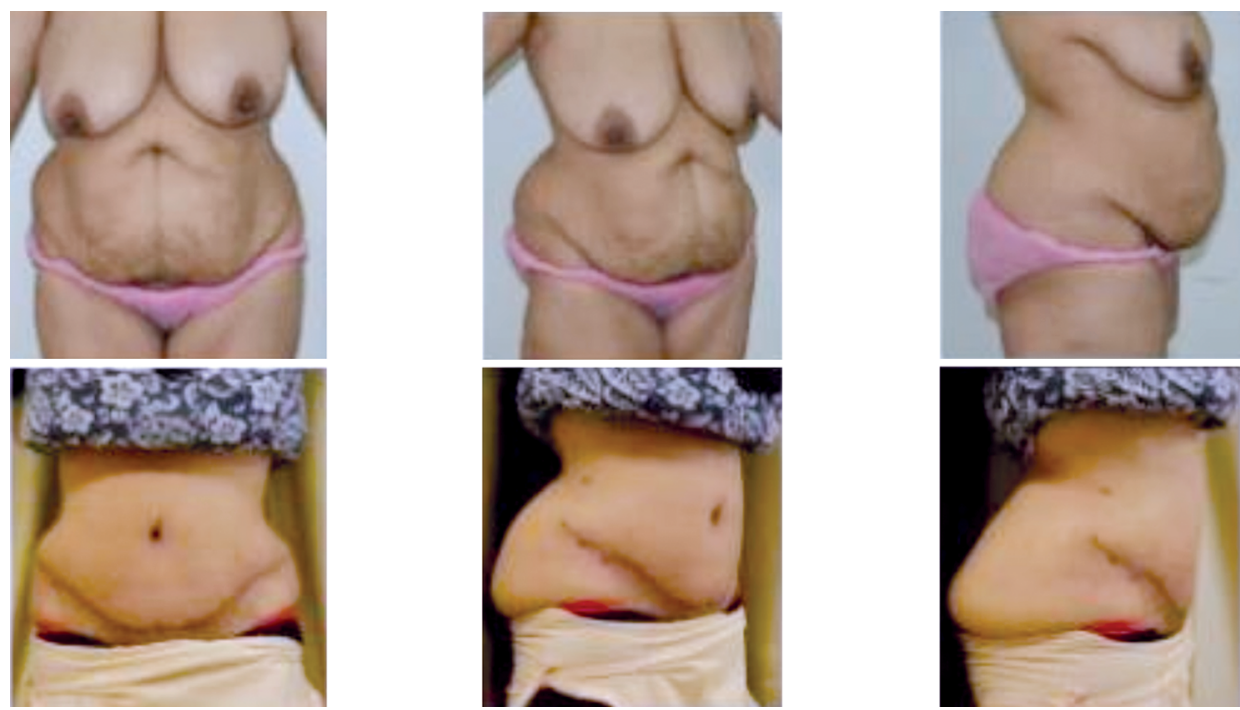

Photo (3): Case 2: Photos before and two months after abdominoplasty using myofascial triangular plication.
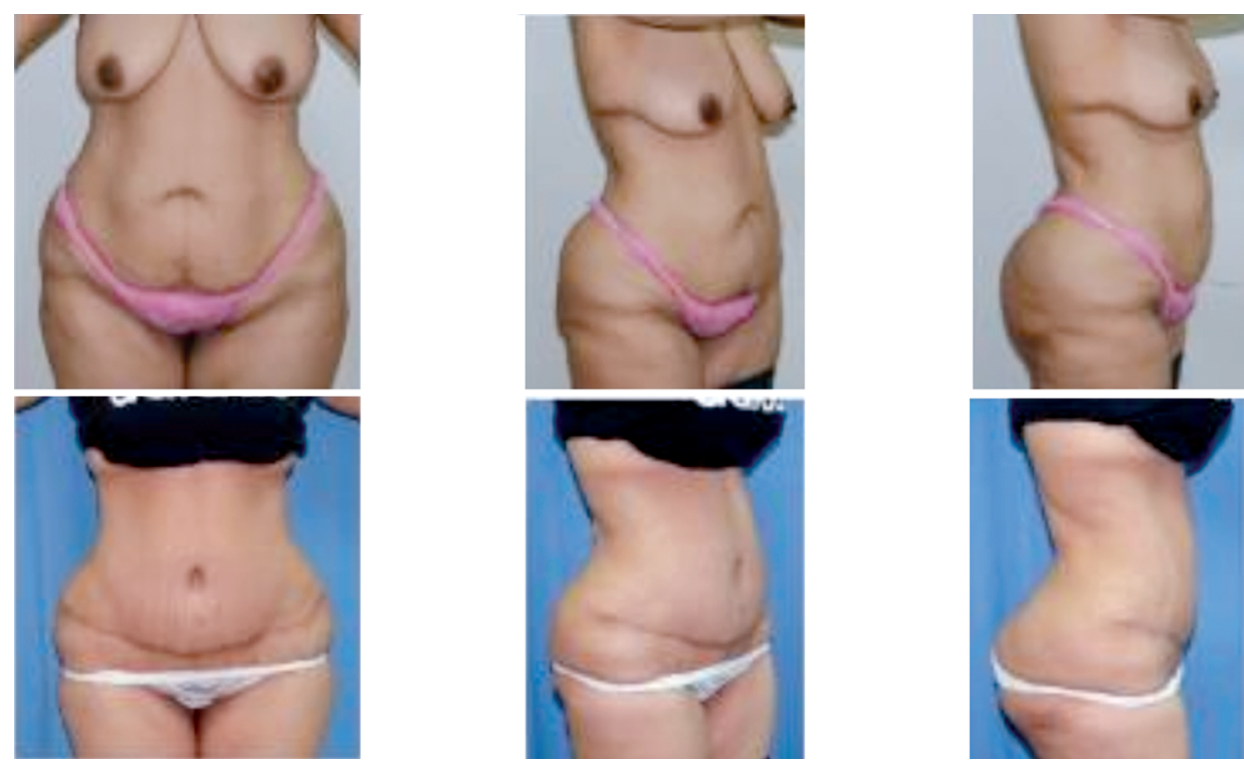

Photo (4): Case 3: Photos before and 4 months after abdominoplasty using myofascial triangular plication. 


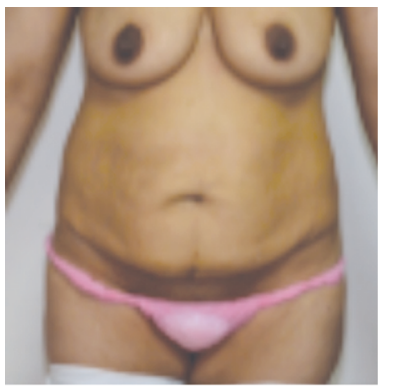

(A)
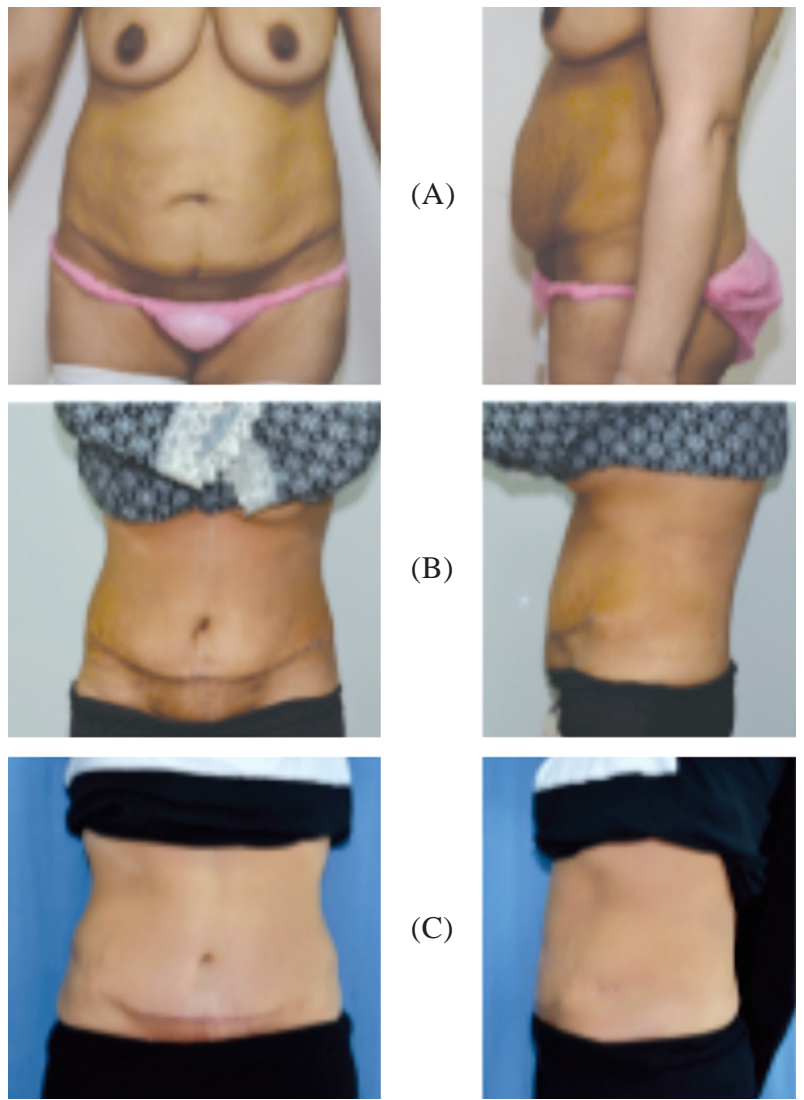

Photo (5): Case 4: (A) before abdominoplasty using the triangular plication (B) six months after abdominoplasty using the triangular plication (C) maintained results after 12 months of abdominoplasty using the triangular plication.

\section{DISCUSSION}

Although rectus diastasis is considered a cosmetic disfigurement, its repair improves the functions of the abdominal muscles and the quality of life of many patients [14]. This is because the tight lateral abdominal wall muscles after repair become more efficient as spine stabilizers and improve the chronic pain of the lower back [15].

Most surgeons perform abdominal myofascial plication to strengthen the weakened abdominal wall and to achieve a narrow waistline $[2,3]$. To meet the increasing aesthetic demands of patients, different musculoaponeurotic system plications have been described, including vertical, horizontal, curved, fusiform, "H"-shaped, and the crossbow plication [5-9]. However, to date, there is no general agreement among plastic surgeons about the best technique to be used [10], leading abdominoplasty to become increasingly complicated [1]. This stimulated the authors to offer the "Triangular Technique" as a new simple technique of abdominal myofascial plication.
The triangular technique reduces both the vertical and horizontal abdominal spans, enhances the waistline, with relatively no marked change in the operative time, and achieves high patients' satisfaction and high Global Aesthetic Improvement Scores (GAIS).

The decrease in the longitudinal distance facilitates lowering and descent of the cutaneous flap without tension. And the slimmer waistline that was obvious during the procedure was maintained later in the postoperative time. There was no marked change in the duration of the triangular plication compared to the duration usually consumed by the authors to perform the traditional midline plication in their hands.

The Rectus sheath plication technique is not standardized and the size and type of sutures to be used are still debatable [16-19]. However, the interrupted triangular suture described by Ferreira et al., [12] is proved to be an effective option for shortening the vertical length of the myoaponeurotic layer. In this triangular myofascial plication technique, the authors used the triangular mattress suture, as required, to do the supra-umbilical part of our triangular plication to shorten the vertical length and proper positioning of the umbilicus.

The traditional vertical rectus plication may be combined selectively with transverse plication to reduce the vertical bulge and improve aesthetic outcomes. This is useful for massive weight loss patients and patients with pronounced diastasis of the recti after pregnancy [20]. However, the levels of the transverse plications are not standardized and differ from one technique to another making many of these techniques complicated with the limitations related to each level e.g., the risk to sequestrate the structure around the inguinal region with transverse plication in the lower abdomen inferior to the level of ASIS, as in crossbow technique, or the excessive lateral dissection for horizontal plications higher in the abdomen.

Our simple triangular plication technique produces the same effect of combined vertical and horizontal plication in reducing both vertical and horizontal lengths and the plication lays above the level of ASIS far from the inguinal region, and centripetal plication forces towards the umbilicus with no excessive lateral dissection required above the umbilicus.

Some surgeons use prosthetic mesh for wide diastasis of the recti to enforce the repair. However, there is no agreement regarding when to use a prosthetic mesh if there is no hernia, and the extra 
operating room time, additional expenses, and potential for mesh-related complications must be considered [21]. The authors in this study did not use a mesh in any of the cases, as the myoaponeurotic layer was well-toned after plication. However, a longer follow-up is required to evaluate the longterm outcomes.

To evaluate the safety of abdominal myofascial repair techniques, researchers in different studies focused on the changes in the intra-abdominal pressure and the ventilatory function [22-24]. As the patient's safety comes first, this study also monitored the changes in peak airway pressure, and the changes of intra-bladder pressure as simple and practical methods of these pressure changes before and after plication. However, the changes of these pressures after triangular myofascial plication were tolerated clinically by almost all patients of this study, 2 weeks postoperatively, and no major complications were encountered. This leads us to question whether the monitoring of these pressures is a valid tool for the evaluation of myofascial plication or no?

The change in intra-abdominal pressure and subsequent intrathoracic pressure following abdominoplasty is another debatable point. Where on the one hand, some researchers statistically proved that intra-abdominal pressure increases after abdominoplasty, especially with the manipulation of the musculoaponeurotic layer, with a consequent increase in the intrathoracic pressure which increases the risk of pulmonary complications [25-27]. On the other hand, other researchers claimed they found no significant differences in pulmonary function and Intra-abdominal Pressure after myofascial plication [28,29].

Despite the authors, in this study, excluded smokers, those with altered pulmonary functions, and those with chronic gaseous distension, three of the patients $(36.9 \%)$ complained of tightness, but with no change of oxygen saturation, which improved over two weeks by proper positioning.

The authors' explanation of the discrepancy of tightness between patients, although the intrabladder and the peak airway pressure were increased after plication in all patients, is that the tightness is related to the variable patients' tolerance of abdominal pain and individual pattern of breathing whether abdominal or thoracic and not the mere increase of pressure which was well tolerated clinically by the patients. However, this needs further in-depth research which was beyond this study. And the authors support that in patients with altered pulmonary status (smokers, COPD), per- forming a conservative plication is practical to avoid postoperative respiratory problems.

In 2006, Bragg et al., [30] did their detailed analysis of patients' dissatisfaction following abdominoplasty and found that, even in carefully selected patients for abdominoplasty, and irrelevant to the age, areas of dissatisfaction were identified to be centered on: The dog-ears and the scar, excess residual abdominal skin, sensory changes, and umbilical related problems. Based on those points of patients' dissatisfaction, we designed the postoperative satisfaction survey to assess these points subjectively besides the narrow waistline which was the main concern of most of the patients of this study, and to assess these points objectively by an external observer using the Global Aesthetic Improved Score (GAIS). The survey used in the current study revealed excellent patients' satisfaction responses in $95 \%$ of the patients included in this study, with GAIS 4-5 in 95\% of the cases.

The limitations of this study are the unintended interrupted follow-up and the relatively small number of cases included.

Conclusion: Abdominal triangular plication is a simple technique that improves the trunk contour and narrows the waist-line and facilitates tensionfree closure of abdominoplasty incision with high patients' satisfaction rates. Further study is recommended for longer follow-up, proper assessment of late outcomes, and comparing it to other abdominal myofascial plication techniques.

Acknowledgment: The authors declare that they have no conflict of interest, and the study followed the principles of the declaration of Helsinki.

\section{REFERENCES}

1- Gutowski K.A.: Evidence-Based Medicine: Abdominoplasty. Plast. Reconstr. Surg., 141 (2): 286e-99e, 2018.

2- Netscher D.T., Wigoda P., Spira M. and Peltier M.: Musculoaponeurotic plication in abdominoplasty: How durable are its effects? Aesthetic Plast. Surg., Dec. 19 (6): 5314, 1995 .

3- Nahas F.X., Augusto S.M. and Ghelfond C.: Should diastasis recti be corrected? Aesthetic Plast. Surg., Aug. 21 (4): 285-9, 1997.

4- Nahas F.X. and Ferreira L.M.: Concepts on Correction of the Musculoaponeurotic Layer in Abdominoplasty. Clin. Plast. Surg., Jul. 37 (3): 527-38, 2010.

5- Yousif NJ, Lifchez SD, Nguyen HH. Transverse rectus sheath plication in abdominoplasty. Plast. Reconstr. Surg., Sep. 1; 114 (3): 778-84, 2004.

6- Marques A., Brenda E., Pereira M.D., de Castro M. and Abramo A.C.: Abdominoplasty with two fusiform plications. Aesthetic Plast. Surg., Jun. 20 (3): 249-51, 1996. 
7- Abramo A.C., Casas S.G., Oliveira V.R. and Marques A.: $\mathrm{H}$-Shaped, double-contour plication in abdominoplasty. Aesthetic Plast. Surg., Aug. 23 (4): 260-6, 1999.

8- Sozer S.O. and Agullo F.J.: Triple Plication in Miniabdominoplasty. Aesthetic Plast. Surg., Jun. 30 (3): 263-8, 2006.

9- Soares Filho I.: Abdominal wall treatment with plication using the crossbow technique. Rev. Bras Cir Plástica RBCP - Braz J. Plast. Sugery, 34 (1): 23-30, 2019.

10- Nahas F.X., Faustino L.D. and Ferreira L.M.: Abdominal Wall Plication and Correction of Deformities of the Myoaponeurotic Layer: Focusing on Materials and Techniques Used for Synthesis. Aesthet Surg J., Mar. 14; 39 (Supplement 2): S78-84, 2019.

11- Desie N., Willems A., De laet I., Dits H., Van Regenmortel N., Schoonheydt K., et al.: Intra-abdominal pressure measurement using the FoleyManometer does not increase the risk for urinary tract infection in critically ill patients. Ann. Intensive Care, Dec. 2 (S1): S10, 2012.

12- Ferreira L.M., Castilho H.T., Hochberg J., Ardenghy M., Toledo S.R., Cruz R.G., et al.: Triangular mattress suture in abdominal diastasis to prevent epigastric bulging. Ann. Plast. Surg., Feb. 46 (2): 130-4, 2001.

13- Delorenzi C., Weinberg M, Solish N. and Swift A.: A Multicenter Study of the Efficacy and Safety of Subcutaneous Nonanimal Stabilized Hyaluronie Acid in Aesthetic Facial Contouring: Interim Report: SUBCUTANEOUS RESTYLANE. Dermatol. Surg., Mar. 15; 32 (2): 205-11, 2006.

14- Strigård K., Clay L., Stark B. and Gunnarsson U.: Predictive Factors in the Outcome of Surgical Repair of Abdominal Rectus Diastasis: Plast. Reconstr. Surg. - Glob Open, May 4 (5): e702, 2016.

15- Oneal R.M., Mulka J.P., Shapiro P., Hing D. and Cavaliere C.: Wide abdominal rectus plication abdominoplasty for the treatment of chronic intractable low back pain. Plast. Reconstr. Surg., Jan. 127 (1): 225-31, 2011.

16- Elkhatib H., Buddhavarapu S.R., Henna H. and Kassem W.: Abdominal musculoaponeuretic system: Magnetic resonance imaging evaluation before and after vertical plication of rectus muscle diastasis in conjunction with lipoabdominoplasty. Plast. Reconstr. Surg., Dec. 128 (6): 733e-40e, 2011.

17- Tadiparthi S., Shokrollahi K., Doyle G.S. and Fahmy F.S.: Rectus sheath plication in abdominoplasty: Assessment of its longevity and a review of the literature. J. Plast. Reconstr. Aesthet. Surg., Mar. 65 (3): 328-32, 2012.

18- Ceydeli A., Rucinski J. and Wise L.: Finding the best abdominal closure: An evidence-based review of the literature. Curr. Surg., Mar. 62 (2): 220-5, 2005.
19- Stewart K.J., Stewart D.A., Coghlan B., Harrison D.H., Jones B.M. and Waterhouse N.: Complications of 278 consecutive abdominoplasties. J. Plast. Reconstr. Aesthet. Surg., Nov. 59 (11): 1152-5, 2006.

20- Serra-Renom J-M., Martinez-Teixido L. and Serra-Mestre J-M.: Abdominoplasty with Customized Transverse Musculoaponeurotic Plications: Plast. Reconstr. Surg., Dec. 136 (6): 741e-9e, 2015.

21- Staalesen T., Olsén M.F. and Elander A.: The Effect of Abdominoplasty and Outcome of Rectus Fascia Plication on Health-Related Quality of Life in Post-Bariatric Surgery Patients: Plast. Reconstr. Surg. Dec. 136 (6): 750e-61e, 2015.

22- Al-Basti H.B., El-Khatib H.A., Taha A., Sattar H.A. and Bener A.: Intraabdominal pressure after full abdominoplasty in obese multiparous patients. Plast. Reconstr. Surg., Jun. 113 (7): 2145-50; discussion 2151-2155, 2004.

23- Mahmoud N.A. and Abdel Hamid W.R.: Assessement of the Correction of Rectus Abdominis Muscles Diastasis Using Two Different Techniques. Egypt J. Plast. Reconstr. Surg., Jul. 1; 43 (2): 271-82, 2019.

24- Hassan M.: Modification of Rectal Myofascial Repair in Abdominoplasty for Patients with Widely Divaricated Recti. Egypt J. Plast. Reconstr. Surg., Oct. 1; 43 (3): 53944, 2020.

25- Tercan M., Bekerecioglu M., Dikensoy O., Kocoglu H., Atik B., Isik D., et al.: Effects of abdominoplasty on respiratory functions: A prospective study. Ann. Plast. Surg., Dec. 49 (6): 617-20, 2002.

26- Watson R.A. and Howdieshell T.R.: Abdominal Compartment Syndrome: South Med. J. Apr. 91 (4): 326-32, 1998.

27- Pereira N., Sciaraffia C., Danilla S., Parada F., Asfora C. and Moral C.: Effects of Abdominoplasty on IntraAbdominal Pressure and Pulmonary Function. Aesthet. Surg. J., Jun. 36 (6): 697-702, 2016.

28- Wilhelmsson S., Fagevik Olsén M., Staalesen T., Elander A. and Nygren-Bonnier M.: Abdominal plasty with and without plication-effects on trunk muscles, lung function, and self-rated physical function. J. Plast. Surg. Hand Surg., Jun. 51 (3): 199-204, 2017.

29- Rodrigues M.A., Nahas F.X., Gomes H.C. and Ferreira L.M.: Ventilatory function and intra-abdominal pressure in patients who underwent abdominoplasty with plication of the external oblique aponeurosis. Aesthetic Plast. Surg., Oct. 37 (5): 993-9, 2013.

30- Bragg T.W.H., Jose R.M. and Srivastava S.: Patient satisfaction following abdominoplasty: An NHS experience. J. Plast. Reconstr. Aesthet. Surg., Jan. 60 (1): 758, 2007. 